(1)

CrossMark

\title{
Does bronchoscopy help the diagnosis in COVID-19 infection?
}

\author{
To the Editor:
}

Severe acute respiratory syndrome coronavirus 2 (SARS-CoV-2) is the agent responsible for the recent coronavirus disease 2019 (COVID-19) pandemic. The virus is predominantly spread through large droplets. The clinical features of COVID-19 are varied, ranging from asymptomatic to acute respiratory distress syndrome and multi-organ dysfunction [1].

The diagnosis of COVID-19 is mainly based on typical symptoms, history of exposure to an infected person and bilateral involvement on chest radiographs; it is confirmed by a positive nucleic acid test for SARS-CoV-2 from numerous types of specimens, including oropharyngeal (OP) and nasopharyngeal (NP) swabs, anal swabs, stool, urine and bronchoalveolar lavage fluid (BALF) $[1,2]$. Reverse transcriptase (RT)-PCR that targets the RdRP, N or E genes is the most common method of SARS-CoV-2 detection [3]. OP and NP swabs are the most frequently used samples, but it has been demonstrated that their sensitivity is limited, at $32 \%$ and $63 \%$ respectively, while BALF is reported to be positive in $93 \%$ of patients [4]. However, the role of bronchoscopy in ruling out suspected COVID-19 patients is under debate. According to several guidelines, bronchoscopy is relatively contraindicated, mainly because of its high risk of spreading the infection to the staff involved in the procedure $[5,6]$. It is primarily recommended in immunocompromised patients, if there is the strong suspicion of superinfection or mucus plugging, or in life-saving conditions, and it is not strictly recommended in the COVID-19 diagnostic algorithm. Nevertheless, some cases of negative OP and NP swabs, in which BALF tested positive for SARS-CoV-2 with RT-PCR, have been reported [7, 8].

There are two main problems with negative-swab patients who have computed tomography (CT) scans and a clinical picture suggestive of COVID-19. First, according to the sensitivity of the swabs, misdiagnosing a SARS-CoV-2-positive patient may be a great risk for public health. Secondly, an alternative diagnosis may be required for patient-appropriate treatment.

Here, we report our experience from a COVID-19 hospital in Rome, Italy, where patients with typical symptoms of the disease, suggestive CT scans, three NP/OP-negative swabs performed on consecutive days, and IgG and IgM serology negative for SARS-CoV-2 underwent bronchoscopy with BAL to define the diagnostic issue.

In the period between March 13th and April 30th, 2020, 28 patients (mean \pm sD age $65 \pm 16$ yrs, 16 male and 12 female) with the described characteristics, discussed by the multidisciplinary clinical-radiological team, underwent bronchoscopy with BAL. All the patients performed a non-enhanced chest CT scan in supine position, from the apex to the base of the lungs during inspiratory breath-hold, with a 128-slice scanner. Two radiologists independently evaluated the axial images with a visual score [9]. CT manifestations were characterised by a predominant ground-glass pattern (68\%) mixed with consolidations (32\%), mainly peripheral $(26 \%)$ or combined peripheral and central distributions (72\%), with the bilateral (83\%) and lower lung zones $(93 \%)$ being mostly involved. Lymphadenopathy and pleural effusion were rarely founded ( $0 \%$ and $4 \%$, respectively). According to several recent reports regarding CT findings in COVID-19 patients [10-12], these results were suggestive of COVID-19 in all considered cases.

BALF was analysed for SARS-CoV-2 using RT-PCR and for routinely microbiological examination. In the BALF, the RT-PCR for SARS-CoV-2 was negative for all three detectable genes in all 28 patients. Microbiological examination was negative in 15 (53.6\%) patients. Of the other 13 patients, six had galactomannans $>0.8$, one patient had galactomannans between 0.5 and 0.8 , four patients were positive at

@ERSpublications

This study evaluates the real value of using bronchoscopy to exclude the diagnosis of COVID-19 in patients with multiple negative nasal/oropharyngeal swabs https://bit.ly/2zmSgE8

Cite this article as: Ora J, Puxeddu E, Cavalli F, et al. Does bronchoscopy help the diagnosis in COVID19 infection? Eur Respir J 2020; 56: 2001619 [https://doi.org/10.1183/13993003.01619-2020]. 
microbiological culture for Candida albicans, two patients were positive for Pneumocystis jirovecii, one patient was positive for Candida glabrata, one patient was positive for for Streptococcus pneumoniae, two patients were positive for Staphylococcus epidermidis, one patient was positive for Klebsiella pneumoniae and one patient was positive for Enterococcus faecium. The final diagnosis was pneumonia in 22 patients (no specific agent in 11 patients, five of which were immunocompromised; specific agent in 11 patients, four of which were immunocompromised), heart failure in three patients, exacerbation of interstitial lung disease in two patients and an acute respiratory distress syndrome in one patient. Antibiotic therapy was modified in 13 patients according to the microbiological findings.

In these pandemic times, the low sensitivity of RT-PCR can miss many diagnoses of COVID-19, representing a far too high risk for infection transmission. On the other hand, even if CT scan has a higher sensitivity (97\%), its lower specificity (25\%) [2] makes alternative diagnoses more likely and bronchoscopy with BALF is the recommend procedure to rule out any doubts.

Our findings suggest that three negative swabs performed on three different days and a negative serology are sufficient to rule out COVID-19, even in patients with highly suggestive CT scans and clinical features compatible with the disease. In contrast with other reports $[7,8]$, we performed three OP/NP swabs and serology tests before performing the bronchoscopy, instead of just one or two OP/NP swabs. This allowed us to detect patients who had positive results at the third swab or at serology, avoiding unnecessary procedures, and therefore reducing the risk of transmission to healthcare workers.

Bronchoscopies were performed with disposable devices and with the recommended personal protective equipment [6]. Even if our results are very reassuring about the safety of this procedure in patients with three negative swabs and negative serology, the risk to the staff still remains high and we strongly suggest that all of the recommended precautions are maintained to minimise the risk of possible disease transmission.

Interestingly, BALF was positive for other pathogens in $46 \%$ of patients, reinforcing its role in finding alternative diagnoses.

In conclusion, our findings demonstrate that three negative swabs along with negative antibodies, despite a suggestive CT scan, can safely rule out the SARS-CoV-2 infection in suspected patients, hence allowing commencement of an alternative diagnosis process.

Bronchoscopy should not be used for the confirmation of SARS-CoV-2 infection alone, but it can be very useful in resolving diagnostic complexity.

Josuel Ora ${ }^{1}$, Ermanno Puxeddu ${ }^{1,2}$, Francesco Cavalli ${ }^{1}$, Federica Maria Giorgino ${ }^{1}$, Andrea Girolami ${ }^{1}$, Marcello Chiocchi ${ }^{3}$, Giaunluigi Sergiacomi ${ }^{3}$, Massimo Federici ${ }^{4}$ and Paola Rogliani $\oslash^{1,2}$

${ }^{1}$ Division of Respiratory Medicine, University Hospital Tor Vergata, Rome, Italy. ${ }^{2}$ Dept of Experimental Medicine, University of Rome Tor Vergata, Rome, Italy. ${ }^{3}$ Dept of Diagnostic Imaging, Molecular Imaging, Interventional Radiology and Radiotherapy, University of Rome Tor Vergata, Rome, Italy. ${ }^{4}$ Dept of Systems Medicine, University of Rome Tor Vergata, Rome, Italy.

Correspondence: Paola Rogliani, Dept of Experimental Medicine, University of Rome Tor Vergata, Via Montpellier 1, 00133, Roma, Italy. E-mail: paola.rogliani@uniroma2.it

Received: 6 May 2020 | Accepted after revision: 3 June 2020

Author contribution: Drafting the article or revising it critically for important intellectual content: J. Ora, E. Puxeddu, F. Cavalli, F.M. Giorgino, A. Girolami, M. Chiocchi, G. Sergiacomi, M. Federici and P. Rogliani. Visual scoring was performed independently by two radiologists: G. Sergiacomi and M. Chiocchi. Final approval of the version to be published: J. Ora, E. Puxeddu, F. Cavalli, F.M. Giorgino, A. Girolami, M. Chiocchi, G. Sergiacomi, M. Federici and P. Rogliani. Agreement to be accountable for all aspects of the work in ensuring that questions related to the accuracy or integrity of any part of the work are appropriately investigated and resolved: J. Ora, E. Puxeddu, F. Cavalli, F.M. Giorgino, A. Girolami, M. Chiocchi, G. Sergiacomi, M. Federici and P. Rogliani.

Conflict of interest: None declared.

Support statement: This study was supported by institutional funding (University of Rome "Tor Vergata”, Rome, Italy).

\section{References}

1 Guan W-J, Ni ZY, Hu Y, et al. Clinical characteristics of coronavirus disease 2019 in China. N Engl J Med 2020; 382: $1708-1720$.

2 Ai T, Yang Z, Hou H, et al. Correlation of chest CT and RT-PCR testing in coronavirus disease 2019 (COVID-19) in China: a report of 1014 cases. Radiology 2020; 296: E32-E40.

3 Interim Guidelines for collecting, handling, and testing clinical specimens from patients under investigation (PUIs) for 2019 novel coronavirus (2019-nCoV). https://www.cdc.gov/coronavirus/2019-nCoV/lab/guidelinesclinical-specimens.html Date last updated: 22 May 2020. Date last accessed: 27 June 2020. 
4 Wang X, Tan L, Wang X, et al. Comparison of nasopharyngeal and oropharyngeal swabs for SARS-CoV-2 detection in 353 patients received tests with both specimens simultaneously. Int J Infect Dis 2020; 94: 107-109.

5 Lentz RJ, Colt H. Summarizing societal guidelines regarding bronchoscopy during the COVID-19 pandemic. Respirology 2020; 25: 574-577.

6 Wahidi MM, Lamb C, Murgu S, et al. American Association for Bronchology and Interventional Pulmonology (AABIP) statement on the use of bronchoscopy and respiratory specimen collection in patients with suspected or confirmed COVID-19 infection. J Bronchol Interv Pulmonol 2020; in press [https://doi.org/10.1097/LBR. 0000000000000681].

7 Tan FR, Qiu YL, Xu Z. [Bronchoalveolar lavage fluid was used to diagnose two cases of 2019-nCoV infection]. Zhonghua Jie He He Hu Xi Za Zhi 2020; 43: 337-339.

8 Winichakoon P, Chaiwarith R, Liwsrisakun C, et al. Negative nasopharyngeal and oropharyngeal swabs do not rule out COVID-19. J Clin Microbiol 2020; 58: e00297-20.

9 Yuan M, Yin W, Tao Z, et al. Association of radiologic findings with mortality of patients infected with 2019 novel coronavirus in Wuhan, China. PLoS ONE 2020; 15: e0230548.

10 Shi H, Han X, Zheng C. Evolution of CT manifestations in a patient recovered from 2019 novel coronavirus (2019-nCoV) pneumonia in Wuhan, China. Radiology 2020; 295: 20.

11 Fang Y, Zhang H, Xu Y, et al. CT manifestations of two cases of 2019 novel coronavirus (2019-nCoV) Pneumonia. Radiology 2020; 295: 208-209.

12 Song F, Shi N, Shan F, et al. Emerging 2019 novel coronavirus (2019-nCoV) pneumonia. Radiology 2020; 295: $210-217$ 